\title{
Totally Robotic Esophagectomy
}

\author{
Chang Hyun Kang, M.D., Ph.D. \\ Department of Thoracic and Cardiovascular Surgery, Seoul National University Hospital, Seoul National University College of Medicine, Seoul, Korea
}

\section{ARTICLE INFO}

Received June 22, 2021

Accepted July 27, 2021

Corresponding author

Chan Hyun Kang

Tel 82-2-2072-3010

Fax 82-2-764-3664

E-mail chkang@snu.ac.kr

ORCID

https://orcid.org/0000-0002-1612-1937
Totally robotic esophagectomy is performed using a robotic technique without additional thoracoscopy or laparoscopy. However, most robotic esophagectomies are currently performed in a hybrid form combining robotic and other endoscopic techniques. Laparoscopic stomach mobilization and thoracoscopic esophagogastric anastomosis are commonly used methods in robotic esophagectomy. In this paper, totally robotic esophagectomy without thoracoscopic or laparoscopic assistance is presented.

Keywords: Robotic surgical procedure, Esophagus neoplasms, Esophagectomy, Minimally invasive surgery

\section{Introduction}

Minimally invasive esophagectomy (MIE) has become a standard operative strategy for esophageal cancer. Fewer postoperative complications and early recovery have been documented in many studies [1-9]. Robotic esophagectomy has been performed as an alternative surgical procedure to conventional thoraco-laparoscopic MIE. Robotic esophagectomy also showed early outcomes comparable to those of thoraco-laparoscopic MIE in previous studies [10-18]. However, most of the surgical procedures in those studies were not completely performed using robotic esophagectomy, but were partly performed using laparoscopy, laparotomy, or thoracotomy. Some parts of the operation, including anastomosis and graft formation, have been selectively performed using thoracoscopic or laparoscopic techniques. Therefore, totally robotic esophagectomy (TRE) has been reported in only a few papers. At the author's institution, TRE has been performed since 2008 and it is currently the standard surgical treatment for esophageal cancer. Therefore, this paper presents the surgical technique in detail, along with a brief summary of the surgical outcomes at the Seoul National University Hospital.

\section{Abdominal phase}

\section{Ports and arms}

Generally, 4 robotic ports are made for the abdominal robotic procedure (Fig. 1A). Three ports are made using an 8 - $\mathrm{mm}$ robotic trocar, and the other one is made using a glove port (NELIS, Bucheon, Korea). Usually, a 2-cm port for arm no. 2 is made first in the periumbilical area. This port is mainly used for the robotic camera and the assis-
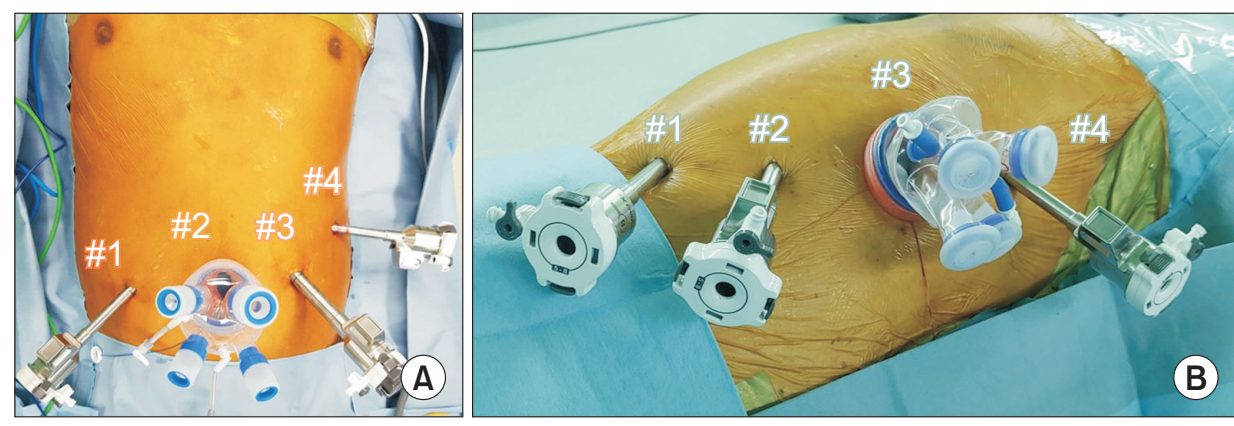

Fig. 1. Port placement for the abdominal (A) and thoracic (B) procedures. The labeled numbers represent the robotic arm number. The detailed location is described in the main text. Robotic staplers are inserted in arm no. 3. Therefore a $12-\mathrm{mm}$ port is necessary for arm no. 3. An assistant surgeon can share the glove port with the robot, and an additional assistant port is not necessary.

Copyright (C) 2021, The Korean Society for Thoracic and Cardiovascular Surgery

c) (i) (-) This is an Open Access article distributed under the terms of the Creative Commons Attribution Non-Commercial License (http://creativecommons.org/licenses/ by-nc/4.0) which permits unrestricted non-commercial use, distribution, and reproduction in any medium, provided the original work is properly cited. 
tant surgeon's work. The port for arm no. 1 is made $10 \mathrm{~cm}$ lateral to the periumbilical port. The port for arm no. 3 is made $7.5 \mathrm{~cm}$ lateral to the periumbilical port and the port for arm no. 4 is made in the right upward area $7.5 \mathrm{~cm}$ lateral to the first right port. The devices used for each trocar are listed in Table 1.

\section{Liver retraction}

Liver retraction is usually performed by Prolene suture traction. Prolene (2-0) with a straight needle is passed just below the xiphoid process. The Prolene is sutured to the crus of the diaphragm and again passed out to the right side of the abdominal wall. The suture is tied outside the abdominal wall. The suture can effectively hold up the right lobe of the liver. This simple procedure suffices for the abdominal procedures described below. I do not use a special liver retractor (Fig. 2A).

\section{Abdominal lymph node dissection}

The dissection of lymph nodes around the celiac axis is

Table 1. The robotic devices used in each arm during abdominal and thoracic procedures

\begin{tabular}{ccl}
\hline Location & Port no. & \multicolumn{1}{c}{ Robotic arms } \\
\hline Abdomen & Arm 1 & Maryland forceps \\
& Arm 2 & Camera, SureForm stapler ${ }^{\mathrm{a})}$ \\
& Arm 3 & Scissors, energy device, needle driver, camera ${ }^{\mathrm{a}}$ ) \\
& Arm 4 & Force bipolar forceps \\
\multirow{4}{*}{ Thorax } & Arm 1 & Maryland forceps \\
& Arm 2 & Camera \\
& Arm 3 & Scissors, energy device, needle driver, \\
& & SureForm stapler \\
& Arm 4 & Force bipolar forceps \\
\hline
\end{tabular}

a) The camera and staplers can be switched with each other during graft stapling. crucial for the staging and treatment of esophageal cancer. For complete dissection of the lymph nodes, the lesser omentum is opened widely from the right gastric artery to the hiatus. The dissection usually starts from lymph node station no. 18 (the common hepatic artery lymph node) and moves through lymph node station no. 17 (the perigastric lymph node) to lymph node station no. 19 (the splenic artery lymph node). The celiac axis lymph nodes (lymph node station no. 20) can also be removed. However, too deep a dissection along the cisterna chyli increases the risk of chyloperitoneum. If the thoracic duct will be ligated in the chest, the surgeon should remember that the risk of chyloperitoneum increases if radical dissection around the cisterna chyli is performed.

\section{Greater omentum division}

The greater omentum is generally divided using an energy device (a Harmonic scalpel or Synchroseal is preferred). Usually, a $5-\mathrm{cm}$ distance is maintained from both gastroepiploic arteries. It is important to maintain this distance along the whole length of the greater omentum. In some patients, a direct connection between the right and left gastroepiploic artery does not exist. In that case, the preserved greater omentum can serve as a collateral flow pathway from the right gastroepiploic artery to the fundus. The Kocher maneuver can be performed, and the first and second portions of the duodenum should be completely mobilized. The Kocher maneuver is not necessary in some cases of thoracic anastomosis. However, when the surgeon plans to perform cervical anastomosis, it is safe to perform the Kocher maneuver routinely. It can provide several additional centimeters of conduit.

\section{Pyloromyotomy}

Pyloromyotomy can be performed by fine dissection us-
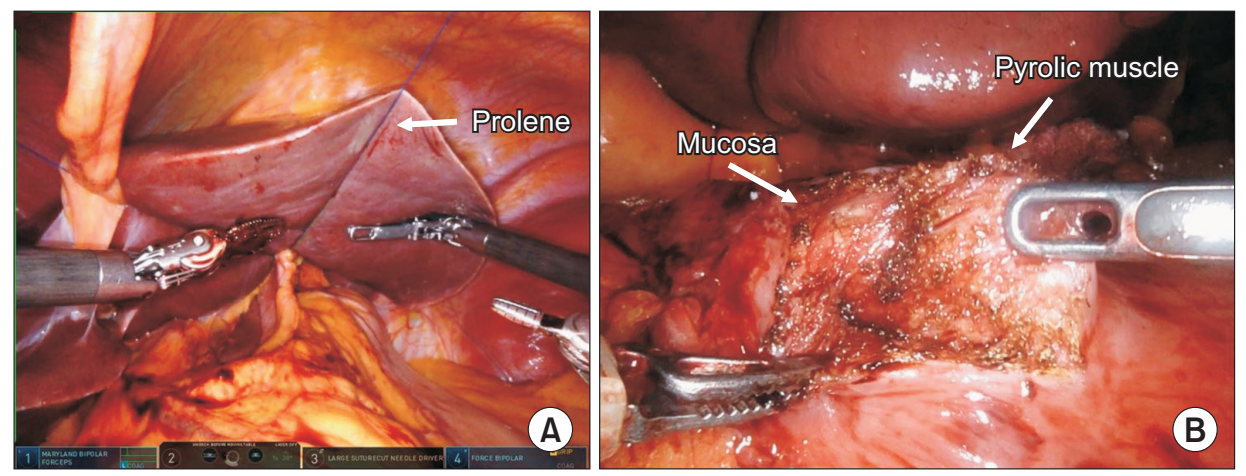

Fig. 2. (A) Liver retraction can be performed by hanging the liver on Prolene string. This procedure makes a liver retractor unnecessary. (B) Pyloromyotomy can be performed by fine division of the pyloric muscle. Making the acute angle of the mucosa into a blunt angle between the stomach and duodenum is a key point for successful pyloromyotomy. 
ing robotic scissors. Coagulation perpendicular to the pyloric muscle can divide the pyloric ring up to the level of submucosal tissue. The power of monopolar coagulation should be less than 2 to avoid accidentally damaging the submucosal area. The divided muscle fibers should be partially detached from the submucosal tissue to straighten the angle of the pyloric mucosal fold (Fig. 2B). The serosal defect is usually covered by the greater omentum using V-Lock (Medtronic, Dublin, Ireland) continuous sutures.

\section{Graft formation}

When the stomach mobilization procedure is completed, the robotic camera can be changed to port no. 3. After the right gastric artery is divided and clipped, the stomach graft can be made by serial stapling with SureForm staplers. I prefer to use $60-\mathrm{mm}$-sized green staplers to divide the stomach (Fig. 3A). Generally, a long graft with a $5-\mathrm{cm}$ width is my preferred method. I routinely check the graft flow using the Firefly system after indocyanine green (ICG) injection (Fig. 3B). When selecting an adequate place for anastomosis, I usually check 2 points: the flow along the marginal artery in the fundus and the serosal uptake of ICG. If the distal fundus does not fulfill those criteria, I usually cut and remove the area with insufficient flow.

\section{Thoracic phase}

\section{Ports and arms}

Four ports are made for thoracic procedures with the same robotic trocars and glove port used in the abdominal procedure. A port for arm no. 1 is made in the 11th intercostal space at the scapular tip level. A port for arm no. 2 is made $7.5 \mathrm{~cm}$ down the right side from port no. 1, and usually at the ninth intercostal space. This port is used for the robotic camera. A port for arm no. 3 is made $7.5 \mathrm{~cm}$ to the right, lateral to port no. 2 with a $3-\mathrm{cm}$ size, and a glove port is inserted into this port. This port is shared by the robot and the assistant surgeon. A port for arm no. 4 is made $7.5 \mathrm{~cm}$ to the right side of port no. 3 (Fig. 1B).

\section{Upper mediastinum and dissection of lymph nodes along the right recurrent laryngeal nerve}

As the first step of esophagectomy, the azygos vein is always divided using Hem-o-Lok clips. The right bronchial artery is also ligated during that procedure. After exposing the upper mediastinum, lymph node dissection along the upper thoracic esophagus (lymph node station no. $8 \mathrm{U}$ ) is performed.

After dividing the mediastinal pleura in the upper mediastinum, lymph node dissection along the right recurrent laryngeal nerve (RLN) can be performed. The proximal part of the right RLN can be identified just below the subclavian artery. Next, the tissues covering the RLN should be completely removed along the right RLN (lymph node station 160RecR in the Japanese Esophageal Society lymph node classification). The limit of the upper end of the dissection is important. The dissection can be performed up to the level of the inferior thyroidal artery and the lower pole of the thyroid gland (Fig. 4A). Therefore, dissection of the cervical para-esophageal lymph node can be performed at this stage (lymph node station no. 1R).

\section{Dissection of lymph nodes along the left recurrent laryngeal nerve}

Left RLN dissection can be performed through a retro-esophageal approach or a pre-esophageal approach. I prefer to use the pre-esophageal approach because most lymphatic tissue is located on the medial side of the left
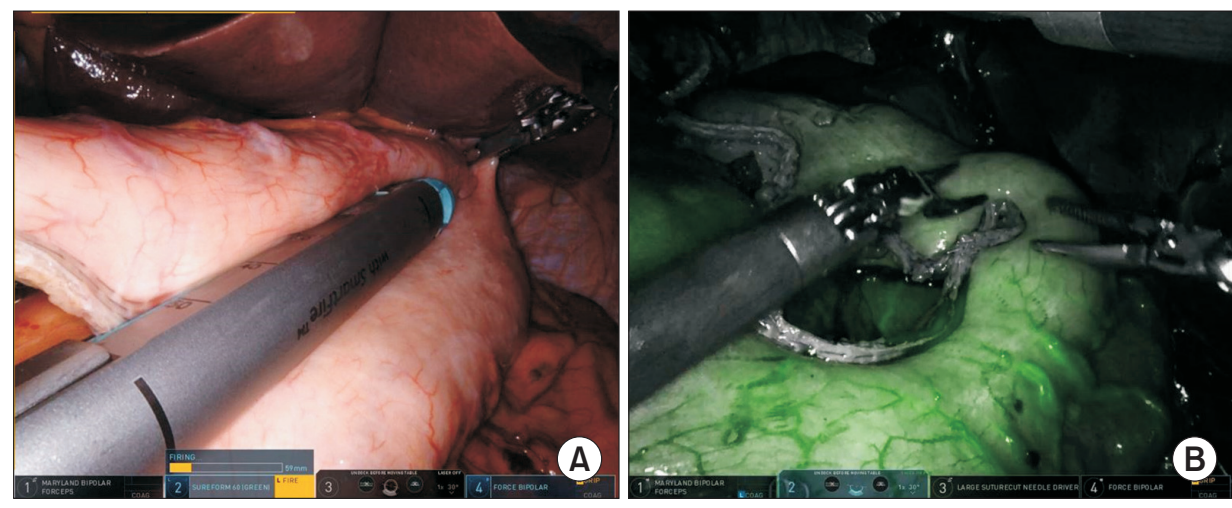

Fig. 3. (A) Intracorporeal graft formation using a robotic SureForm stapler. The entire graft formation procedure can be completed without additional laparotomy. Robotic staplers increase the degree of freedom of the operating surgeon to determine the shape and the length of the stomach graft. (B) Graft blood flow is always checked by indocyanine green infusion after finishing graft formation. Identifying and excluding any ischemic area is important for preventing anastomotic leakage. 
RLN. Therefore, retro-esophageal mobilization and exposure of the lateral side of the left RLN are not necessary (Fig. 4B). The other advantage of the pre-esophageal approach is better exposure of the lower cervical area. Because the esophagus is located away from the trachea in the pre-esophageal approach, exposure of the lower cervical area is more comfortable than using the retro-esophageal approach. Usually, the left RLN can be identified at the aortic arch level because the lymphatic tissue in this area is thinner than in other areas. Dissection along the left RLN can be performed in the area between the aortic arch and the left main bronchus (lymph node station no. 10L, 106TBL in the Japanese Esophageal Society lymph node classification) and along the left RLN (lymph node station no. 4L and 2L, 106RecL in the Japanese Esophageal Society lymph node classification). The upper limit of the dissection is the same as on the right side (inferior thyroidal artery and lower pole of the thyroid gland), and the left cervical para-esophageal lymph nodes can be removed (lymph node station no. 1L).

\section{Lower mediastinum and thoracic duct control}

Mid and lower-thoracic esophageal dissection is usually performed using an energy device. The vagus nerve is usually divided at the level below the pulmonary branches. The subcarinal (lymph node station no. 7), mid-esophageal (lymph node station no. 8M), lower esophageal (lymph node station no. $8 \mathrm{~L}$ ), both pulmonary ligaments (lymph node station no. 9R and 9L), and diaphragmatic lymph nodes (lymph node station no. 15) are removed at this stage. The thoracic duct is usually not divided and paraaortic tissues are preserved to avoid injuring the thoracic duct. However, if a safe radial margin cannot be achieved with thoracic duct excision or branches of the thoracic duct are injured during dissection, the duct is removed completely from the hiatus to the thoracic inlet level.

\section{Gastric pull-up and anastomosis}

After finishing the esophageal dissection, the gastric graft can be pulled up and esophagogastric anastomosis can be made at the thoracic inlet level. I prefer to use triangular linear stapling with a robotic SureForm stapler. I usually use $60-\mathrm{mm}$ green staples for this anastomosis (Fig. $5 \mathrm{~A})$. The goal is to make the diameter of the anastomosis large enough to prevent anastomosis stricture. After finishing the stapling, seromuscular suturing can be performed using V-lock continuous sutures (Fig. 5B).

\section{Outcomes}

At the author's institution, TRE was initiated in 2008. However, the main robotic esophagectomy procedure in
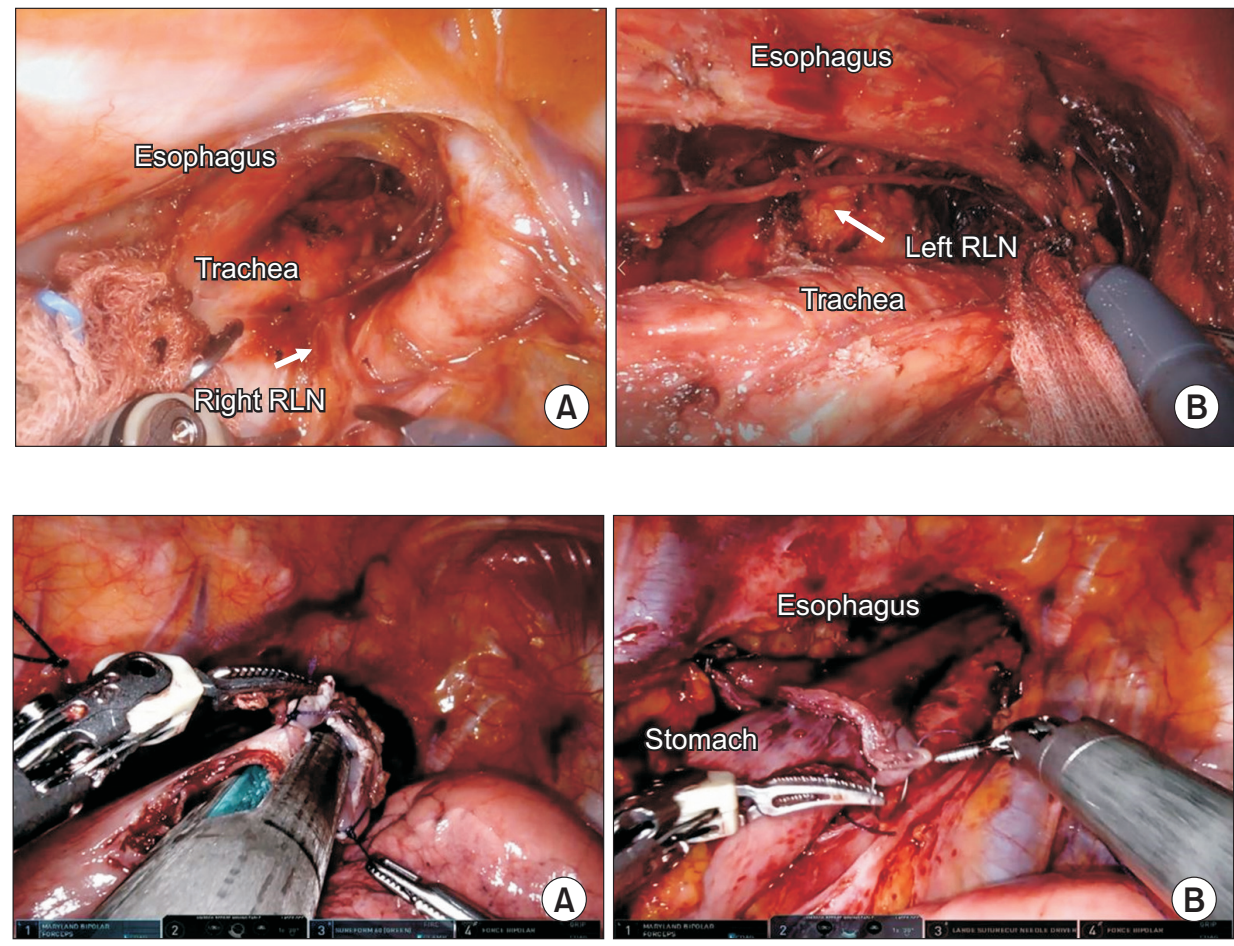

Fig. 4. (A) Lymph node dissection along the right recurrent laryngeal nerve (RLN). All lymphatic tissues between the right RLN and esophagus can be removed. The dissection can reach the lower pole of the thyroid gland. (B) Lymph node dissection along the left RLN by the pre-esophageal approach. The range of dissection is same as on the right side. The dissection can be performed completely in the upper mediastinum and the lower neck.

Fig. 5. Robotic triangular stapling with SureForm staplers. (A) 60-mm SureForm staplers are used to make a large anastomosis circumference. (B) The shape of the anastomosis after finishing anterior wall stapling. Wide stretching of the esophageal and gastric walls can be seen. Robotic triangular stapling reduces the anastomosis stricture and prevents postoperative difficulty in swallowing. 
the early period was a hybrid form with open laparotomy or laparoscopy. The totally robotic approach became the main procedure in 2015, and almost all robotic esophagectomies are now performed using TRE. We have performed 250 TRE cases since 2008. The early outcomes of TRE were favorable, with a $0.4 \% 30$-day mortality and $0.8 \% 90$-day mortality rate. Anastomotic leakage and pulmonary complications occurred in $4.7 \%$ and $9.8 \%$ of patients, respectively. The overall 5 -year survival rate of TRE is $67.0 \%$.

\section{Discussion}

MIE has become one of the standard operations in the treatment of esophageal cancer. In several randomized controlled trials, MIE reduced pulmonary complications, enhanced recovery, and improved quality of life [1-3]. Most centers throughout the world currently perform MIE in significant numbers of patients, and the number of MIEs performed is increasing worldwide [7,19-21]. The main technique in MIE is a combination of laparoscopic and thoracoscopic techniques. However, there are hybrid forms of MIE using utility thoracotomy and laparotomy for intrathoracic anastomosis and gastric graft formation [22-25]. Currently, there is no clear definition of MIE, and the term "MIE" is used in the literature to describe a wide variety of operations.

The same phenomenon has also occurred in robotic surgery. Technically, robotic esophagectomy should be performed using the robot in both the chest and the abdomen. However, many different types of robotic esophagectomy have been described in the literature. The most common type of robotic esophagectomy is a hybrid form with robotic thoracic procedures and laparoscopic abdominal procedures $[3,26,27]$. Because of the difficulty of thoracoscopic esophagectomy and mediastinal lymph node dissection, the robotic technique has mainly been used for thoracic procedures. However, laparoscopic abdominal procedures have been preferred because of the relative ease of the abdominal procedure. Here, a question can be raised regarding whether the hybrid robotic esophagectomy procedure can truly reflect the advantages of robotic technology.

Robotic technology was developed to improve minimally invasive surgery. Several technological developments have been documented as unique advantages of robotic surgery, including improved camera resolution, a 3-dimensional surgical view, full control of the robotic arms by the surgeon, free articulation of the robotic instruments, and many specialized devices dedicated to the robotic system. The benefits of robots can be maximized by fully adapting and maximally utilizing these technological improvements. However, the hybrid form of robotic surgery, which uses laparoscopic and thoracoscopic techniques, cannot fully utilize the robotic system. Therefore, a clear definition of robotic esophagectomy is required and comparative studies between different surgical techniques should be performed. However, it is anticipated that those heterogeneous technical variations will be unified into totally robotic surgery in concordance with advances in robotic technology.

A randomized controlled study comparing robotic and open esophagectomy was recently published [3]. In this trial, robotic esophagectomy improved early postoperative outcomes, with fewer pulmonary complications and improved quality of life. Nonetheless, when compared to thoraco-laparoscopic MIE, the advantages of robotic esophagectomy are unclear. However, several advantages have been suggested in previous studies. The first advantage of robotic esophagectomy is the quality of mediastinal lymph node dissection, especially lymph node dissection around the RLN. The lymph nodes around the RLN are the lymph node stations to which esophageal cancer most commonly metastasizes [28]. They are numbered as $2 \mathrm{R}, 2 \mathrm{~L}, 4 \mathrm{~L}$, and $10 \mathrm{~L}$ in the American Joint Committee on Cancer system [28], and as 106RecR, 106RecL, and 106 Tbl in the Japanese Esophageal Society lymph node map [29]. The dissection of those lymph nodes is technically challenging because of the danger of RLN injury. Nerve injury poses a considerable risk of inducing aspiration pneumonia and deteriorating patients' quality of life. Park et al. [13] compared the quality of mediastinal lymph node dissection, and reported that the number of the dissected lymph nodes along the RLN was higher in robotic esophagectomy than in thoracoscopic esophagectomy. Since then, several other studies have also confirmed the same results, reporting that robotic surgery showed better performance in dissection along the RLN nodes than thoracoscopic surgery $[12,18,30,31]$. Other researchers reported a reduced incidence of RLN palsy after robotic surgery, which may be another advantage of robotic esophagectomy [32-34]. Zheng et al. [34] also documented a lower incidence of vocal cord palsy in the robotic surgery group in their meta-analysis. The dissection of lymph nodes along both RLNs is the most beneficial aspect of robotic esophagectomy.

In other aspects of esophagectomy, there is not enough evidence to determine whether robotic esophagectomy is better than thoracoscopic or laparoscopic esophagectomy. $\mathrm{Na}$ et al. [11] reported similar numbers of dissected lymph nodes in the abdomen compared to laparotomy. However, studies comparing the quality of abdominal procedures in 
different surgical methods are rare. In terms of the outcomes of anastomosis, the method of robotic anastomosis is quite heterogeneous and the outcomes are not well enough documented for comparison to the thoracoscopic technique. Regarding the respiratory complication rate, the outcomes of robotic surgery and thoracoscopic surgery vary. A meta-analysis paper published by Zheng et al. [34] reported a lower incidence of pulmonary complications in robotic surgery. However, few individual studies have demonstrated a lower incidence of pulmonary complications in robotic surgery. Therefore, those issues should be clarified in future studies.

Long-term survival after MIE has been reported in many studies, and MIE is now generally considered to be comparable to open esophagectomy in terms of long-term survival $[2,35,36]$. However, the long-term survival of patients undergoing robotic esophagectomy has only been reported in a small number of studies in patients with different clinical and pathological stages. Van der Sluis [3] reported comparative outcomes between robotic esophagectomy and open esophagectomy in patients with advanced (stage III and IV) esophageal cancer. The 5-year survival rate of robotic surgery and open surgery was $41 \%$ and $40 \%$, respectively, without a significant difference. Park et al. [13] reported long-term outcomes after robotic and thoracoscopic esophagectomy in patients with various stages of the disease. The 5 -year overall survival rate of robotic and thoracoscopic esophagectomy was $69 \%$ and 59\%, respectively; this difference was not statistically significant. According to currently published studies, the long-term survival of patients undergoing robotic esophagectomy is comparable to that of patients undergoing open or thoracoscopic esophagectomy.

Last, learning curve issues should be addressed. Although the outcomes of robotic surgery have been reported for a long time in the literature, many series included early patients who underwent the procedure during the surgeon's learning period. Therefore, the outcomes of robotic surgery in the early period cannot be compared to the outcomes of established centers. The learning curve of a new surgical method can affect the outcomes of esophagectomy at various levels. Park et al. [37] reported that different numbers of cases were necessary for different surgical procedures. This learning curve effect can be shortened by proper proctorship [38]. The learning curve effect should always be considered when interpreting the results of robotic surgery papers. Many centers are still in the early learning period because robotic surgery has been introduced more recently than open esophagectomy or thora- co-laparoscopic MIE.

\section{Conclusion}

In conclusion, robotic esophagectomy is an evolving technique and the outcomes of the operation have improved over the last decades. However, many heterogeneous surgical techniques are currently performed globally and a direct comparison of the outcomes is difficult. TRE is a method that can standardize the robotic surgical technique and maximize the benefits of the robotic surgical system. This article presented the technical aspects of TRE at the author's institution in detail and reviewed the current status of robotic esophagectomy in the literature.

\section{Conflict of interest}

Prof. Kang is a consultant doctor of Intuitive Surgical Korea Inc. Otherwise, no potential conflict of interest relevant to this article was reported.

\section{ORCID}

Chang Hyun Kang: https://orcid.org/0000-0002-1612-1937

\section{References}

1. Biere SS, van Berge Henegouwen MI, Maas KW, et al. Minimally invasive versus open oesophagectomy for patients with oesophageal cancer: a multicentre, open-label, randomised controlled trial. Lancet 2012;379:1887-92.

2. Mariette C, Markar SR, Dabakuyo-Yonli TS, et al. Hybrid minimally invasive esophagectomy for esophageal cancer. N Engl J Med 2019; 380:152-62.

3. Van der Sluis PC, van der Horst S, May AM, et al. Robot-assisted minimally invasive thoracolaparoscopic esophagectomy versus open transthoracic esophagectomy for resectable esophageal cancer: a randomized controlled trial. Ann Surg 2019;269:621-30.

4. Briez N, Piessen G, Torres F, Lebuffe G, Triboulet JP, Mariette C. Effects of hybrid minimally invasive oesophagectomy on major postoperative pulmonary complications. Br J Surg 2012;99:1547-53.

5. Yoshida N, Yamamoto H, Baba H, et al. Can minimally invasive esophagectomy replace open esophagectomy for esophageal cancer?: latest analysis of 24,233 esophagectomies from the Japanese National Clinical Database. Ann Surg 2020;272:118-24.

6. Booka E, Tsubosa Y, Haneda R, Ishii K. Ability of laparoscopic gastric mobilization to prevent pulmonary complications after open thoracotomy or thoracoscopic esophagectomy for esophageal cancer: a systematic review and meta-analysis. World J Surg 2020;44:980-9. 
7. Takeuchi H, Miyata H, Ozawa S, et al. Comparison of short-term outcomes between open and minimally invasive esophagectomy for esophageal cancer using a nationwide database in Japan. Ann Surg Oncol 2017;24:1821-7.

8. Luketich JD, Alvelo-Rivera M, Buenaventura PO, et al. Minimally invasive esophagectomy: outcomes in 222 patients. Ann Surg 2003; 238:486-95.

9. Luketich JD, Pennathur A, Awais O, et al. Outcomes after minimally invasive esophagectomy: review of over 1000 patients. Ann Surg 2012; 256:95-103.

10. Van Hillegersberg R, Boone J, Draaisma WA, Broeders IA, Giezeman MJ, Borel Rinkes IH. First experience with robot-assisted thoracoscopic esophagolymphadenectomy for esophageal cancer. Surg Endosc 2006;20:1435-9.

11. Na KJ, Park S, Park IK, Kim YT, Kang CH. Outcomes after total robotic esophagectomy for esophageal cancer: a propensity-matched comparison with hybrid robotic esophagectomy. J Thorac Dis 2019; 11:5310-20.

12. Chao YK, Hsieh MJ, Liu YH, Liu HP. Lymph node evaluation in robot-assisted versus video-assisted thoracoscopic esophagectomy for esophageal squamous cell carcinoma: a propensity-matched analysis. World J Surg 2018;42:590-8.

13. Park S, Hwang Y, Lee HJ, Park IK, Kim YT, Kang CH. Comparison of robot-assisted esophagectomy and thoracoscopic esophagectomy in esophageal squamous cell carcinoma. J Thorac Dis 2016;8:285361.

14. Sarkaria IS, Rizk NP. Robotic-assisted minimally invasive esophagectomy: the Ivor Lewis approach. Thorac Surg Clin 2014;24:211-22.

15. Zhang Y, Han Y, Gan Q, et al. Early outcomes of robot-assisted versus thoracoscopic-assisted Ivor Lewis esophagectomy for esophageal cancer: a propensity score-matched study. Ann Surg Oncol 2019;26: 1284-91.

16. Deng HY, Huang WX, Li G, et al. Comparison of short-term outcomes between robot-assisted minimally invasive esophagectomy and video-assisted minimally invasive esophagectomy in treating middle thoracic esophageal cancer. Dis Esophagus 2018;31:doy012.

17. Deng HY, Luo J, Li SX, et al. Does robot-assisted minimally invasive esophagectomy really have the advantage of lymphadenectomy over video-assisted minimally invasive esophagectomy in treating esophageal squamous cell carcinoma?: a propensity score-matched analysis based on short-term outcomes. Dis Esophagus 2019;32: doy 110 .

18. Yang Y, Zhang X, Li B, et al. Short- and mid-term outcomes of robotic versus thoraco-laparoscopic McKeown esophagectomy for squamous cell esophageal cancer: a propensity score-matched study. Dis Esophagus 2020;33:doz080.

19. Sihag S, Kosinski AS, Gaissert HA, Wright CD, Schipper PH. Minimally invasive versus open esophagectomy for esophageal cancer: a comparison of early surgical outcomes from the Society of Thoracic
Surgeons National Database. Ann Thorac Surg 2016;101:1281-9.

20. Seesing MF, Gisbertz SS, Goense L, et al. A propensity score matched analysis of open versus minimally invasive transthoracic esophagectomy in the Netherlands. Ann Surg 2017;266:839-46.

21. Mamidanna R, Bottle A, Aylin P, Faiz O, Hanna GB. Short-term outcomes following open versus minimally invasive esophagectomy for cancer in England: a population-based national study. Ann Surg 2012;255:197-203.

22. Bonavina L, Scolari F, Aiolfi A, et al. Early outcome of thoracoscopic and hybrid esophagectomy: propensity-matched comparative analysis. Surgery 2016;159:1073-81.

23. Ichikawa H, Miyata G, Miyazaki S, et al. Esophagectomy using a thoracoscopic approach with an open laparotomic or hand-assisted laparoscopic abdominal stage for esophageal cancer: analysis of survival and prognostic factors in 315 patients. Ann Surg 2013;257:87385.

24. Fumagalli U, Baiocchi GL, Celotti A, et al. Incidence and treatment of mediastinal leakage after esophagectomy: insights from the multicenter study on mediastinal leaks. World J Gastroenterol 2019;25: 356-66.

25. Mu JW, Gao SG, Xue Q, et al. Updated experiences with minimally invasive McKeown esophagectomy for esophageal cancer. World J Gastroenterol 2015;21:12873-81.

26. Zhang X, Su Y, Yang Y, et al. Robot assisted esophagectomy for esophageal squamous cell carcinoma. J Thorac Dis 2018;10:3767-75.

27. Yun JK, Chong BK, Kim HJ, et al. Comparative outcomes of robot-assisted minimally invasive versus open esophagectomy in patients with esophageal squamous cell carcinoma: a propensity score-weighted analysis. Dis Esophagus 2020;33:doz071.

28. Akutsu Y, Kato K, Igaki H, et al. The prevalence of overall and initial lymph node metastases in clinical T1NO thoracic esophageal cancer: from the results of JCOG0502, a prospective multicenter study. Ann Surg 2016;264:1009-15.

29. Japan Esophageal Society. Japanese Classification of Esophageal Cancer, 11th edition: part II and III. Esophagus 2017;14:37-65.

30. Xu Y, Li XK, Cong ZZ, et al. Long-term outcomes of robotic-assisted versus thoraco-laparoscopic McKeown esophagectomy for esophageal cancer: a propensity score-matched study. Dis Esophagus 2020 Nov 5 [Epub]. https://doi.org/10.1093/dote/doaa114.

31. Duan X, Yue J, Chen C, et al. Lymph node dissection around left recurrent laryngeal nerve: robot-assisted vs. video-assisted McKeown esophagectomy for esophageal squamous cell carcinoma. Surg Endosc 2020 Oct 26 [Epub]. https://doi.org/10.1007/s00464-020-081052 .

32. Suda K, Ishida Y, Kawamura Y, et al. Robot-assisted thoracoscopic lymphadenectomy along the left recurrent laryngeal nerve for esophageal squamous cell carcinoma in the prone position: technical report and short-term outcomes. World J Surg 2012;36:1608-16.

33. Oshikiri T, Goto $\mathrm{H}$, Horikawa $\mathrm{M}$, et al. Incidence of recurrent laryn- 
geal nerve palsy in robot-assisted versus conventional minimally invasive McKeown esophagectomy in prone position: a propensity score-matched study. Ann Surg Oncol 2021 May 25 [Epub]. https:// doi.org/10.1245/s10434-021-10123-w.

34. Zheng C, Li XK, Zhang C, et al. Comparison of short-term clinical outcomes between robot-assisted minimally invasive esophagectomy and video-assisted minimally invasive esophagectomy: a systematic review and meta-analysis. J Thorac Dis 2021;13:708-19.

35. Straatman J, van der Wielen N, Cuesta MA, et al. Minimally invasive versus open esophageal resection: three-year follow-up of the previously reported randomized controlled trial: the TIME trial. Ann Surg 2017;266:232-6.
36. Gottlieb-Vedi E, Kauppila JH, Malietzis G, Nilsson M, Markar SR, Lagergren J. Long-term survival in esophageal cancer after minimally invasive compared to open esophagectomy: a systematic review and meta-analysis. Ann Surg 2019;270:1005-17.

37. Park S, Hyun K, Lee HJ, Park IK, Kim YT, Kang CH. A study of the learning curve for robotic oesophagectomy for oesophageal cancer. Eur J Cardiothorac Surg 2018;53:862-70.

38. Van der Sluis PC, Ruurda JP, van der Horst S, Goense L, van Hillegersberg R. Learning curve for robot-assisted minimally invasive thoracoscopic esophagectomy: results from 312 cases. Ann Thorac Surg 2018;106:264-71. 